\title{
Uma revisão sistemática para identificação das possibilidades de aplicações e das barreiras de adoção da customização em massa na produção de alimentos
}

\author{
A systematic review to identify possible applications and barriers to the adoption of
} mass customization in food production

\author{
Luiz Philipi Calegari ${ }^{1 *}$, Diego de Castro Fettermann ${ }^{1}$ \\ ${ }^{1}$ Universidade Federal de Santa Catarina (UFSC), Departamento de Engenharia de Produção, Trindade, Florianópolis/SC - Brasil
}

\section{*Corresponding Author}

Luiz Philipi Calegari, Universidade Federal de Santa Catarina (UFSC), Departamento de Engenharia de Produção, Rua Ogê Fortkamp, 111, CEP: 88036-610, Trindade, Florianópolis/SC - Brasil, e-mail: luizpcalegari@gmail.com

Cite as: A systematic review to identify possible applications and barriers to the adoption of mass customization in food production. Braz. J. Food Technol., v. 21, e2017096, 2018.

\section{Resumo}

Em um cenário de indústria 4.0 e integração de processos, torna-se necessária a discussão sobre a produção de produtos personalizados em massa ao consumidor. As empresas possuem dificuldades na implementação da estratégia de Customização em Massa (CM), especialmente em empresas produtoras de alimentos, o que pode ser explicado pela falta de estudos sobre fatores que afetam o sucesso da adoção desta estratégia. A partir de uma revisão sistemática da literatura, este artigo possui o objetivo de realizar uma análise bibliométrica com a finalidade de identificar as possibilidades da adoção de CM para produtos alimentícios customizados em massa e, ainda, identificar barreiras relacionadas à implementação da CM. Os resultados apresentam a perecibilidade dos alimentos, a dificuldade no processamento, a quantificação dos valores nutricionais do produto customizado e a complexidade de percepção do valor agregado da customização pelos clientes como barreiras para a implementação no setor alimentício. Os resultados apresentados contribuem para a identificação de oportunidades de novos produtos e também para a reunião de informações que possibilitem um direcionamento da implementação de CM no setor alimentício.

Palavras-chave: Inovações; Personalização; Produtos alimentícios; Processos alimentícios; Bibliometria; Aplicabilidade.

\section{Abstract}

In a 4.0 industry scenario related to process integration, it becomes necessary to discuss the production of mass customized products for consumers. The companies have difficulties in implementing the strategy of mass customization (MC), especially in food processing companies, which can be explained by the lack of studies on factors that affect the success of the adoption of this strategy. As from a systematic literature review, this article aims to carry out a bibliometric analysis with the objective of investigating the possibilities of applying MC for mass customized food products, and identify barriers related to the implementation of MC. The results showed food perishability, processing difficulties, quantification of the nutritional values of the customized product and the complexity of the perception by the clients of the value added by customization as barriers to its implementation in the food sector. The results presented contribute to the identification of opportunities for new products and to the gathering of information that allows for guidance in the implementation of MC in the food sector.

Keywords: Innovation; Personalization; Food products; Food process; Bibliometry; Applicability. 
Uma revisão sistemática para identificação das possibilidades de aplicações e das barreiras de adoção da customização em massa na produção de alimentos

Calegari, L. P.; Fettermann, D. C.

\section{Introdução}

A estratégia de Customização em Massa (CM) (DAVIS, 1987) tem como objetivo o atendimento de demandas específicas dos consumidores (PINE, 1993) a partir da aplicação de tecnologias e sistemas de produção aptos a entregar bens e serviços com eficiência semelhante aos produzidos em massa (TSENG et al., 1996). Aplicações bem-sucedidas da CM podem ser verificadas em empresas das mais variadas áreas de atuação (FOGLIATTO et al., 2012), como nas companhias Bosch, Nokia, Philips e Siemens (LINDEN et al., 2007), setor de serviços (PETERS; SAIDIN, 2000), automobilística (ALFORD et al., 2000; BRABAZON et al., 2010) e móveis modulados (FETTERMANN; ECHEVESTE, 2014). No setor de alimentos é verificada uma maior quantidade de aplicações na área de nutrição personalizada (BOLAND, 2008) e de produção de alimentos (McINTOSH et al., 2010).

Embora se identifiquem diversos estudos sobre o tema na literatura, ainda há dificuldades de compreensão de alguns limites da adoção de CM (PILLER et al., 2005) e da aplicação de seus conceitos nas empresas (FOGLIATTO et al., 2012; FETTERMANN; ECHEVESTE, 2014). Esta dificuldade é mais crítica na indústria alimentícia (FISHER et al., 2005; MATTHEWS et al., 2006; BOLAND, 2006). Esta área apresenta quantidades restritas de estudos com orientações da utilização de CM em alimentos (McINTOSH et al., 2010). Essa menor quantidade de estudos pode ser explicada pelas diferenças nos processos de produção dos alimentos e por características do produto, tais como perecibilidade, restrições de legislação (McINTOSH et al., 2010) e pelas modificações físico-químicas que podem comprometer a eficiência das operações e a qualidade dos produtos (WEDZICHA; ROBERTS, 2006). Somado à escassez de informações que orientem a implementação de CM na indústria alimentícia, verifica-se a necessidade de reunir a literatura sobre este tema, que ainda se encontra dispersa em diferentes áreas de estudo, tais como na identificação das necessidades dos consumidores (ADEIGBE et al., 2015; NAGPAL et al., 2015; WOLF; ZHANG, 2016), nos métodos de processamento (FISHER et al., 2005; MATTHEWS et al., 2011) ou então em estudos vinculados à cadeia de valor (MERTINS et al., 2012; VERDOUW et al., 2014).

Os produtores de alimentos possuem dificuldades de adequar seus processos a técnicas de fabricação flexíveis para a produção de produtos personalizados de forma econômica (COHEN et al., 2009). Apesar dessas dificuldades, ainda se verifica um aumento de customização de produtos alimentícios ofertados no mercado, como pode ser observado em empresas como as redes de franquias Subway, McDonald's e Domino's (NAGPAL et al., 2015; PIZAM, 2007). No Brasil, além das redes internacionais de fast food, também se verifica a disseminação da adoção da estratégia de CM na alimentação por quilo, que permite a customização da refeição pelo cliente. Apesar desse tipo de estratégia estar bastante difundida no país, não se verificam casos difundidos em outras áreas de atuação das empresas. O crescimento da utilização de $\mathrm{CM}$ em produtos alimentícios (NAGPAL et al., 2015) e do desejo de produtos personalizados (BALCOMBE et al, 2016), em conjunto com a falta de estudos e a dificuldade de sua aplicação na área (BOLAND, 2006; McINTOSH et al., 2010; WEDZICHA; ROBERTS, 2006), apresentam-se como uma oportunidade de pesquisa sobre o tema. Por meio de uma revisão de literatura, este artigo tem por objetivo realizar uma análise bibliométrica da literatura já existente sobre CM no setor alimentício, e identificar possibilidades de aplicações e barreiras para implementação de CM na produção de alimentos.

\section{Método de pesquisa}

Para reunir as oportunidades de pesquisa, foi realizada uma revisão sistemática da literatura. A revisão sistemática adota um processo replicável, científico e transparente (COOK et al., 1997), sendo recomendada para reunir e analisar os estudos relevantes sobre o tema (KITCHENHAM, 2004). A utilização desta abordagem de pesquisa garante que o erro sistemático seja limitado, os efeitos casuais sejam reduzidos e a legitimidade da análise de dados seja reforçada (REIM et al., 2015). A pesquisa deste trabalho baseou-se no método desenvolvido por Kitchenham (2004), amplamente utilizado na literatura (KITCHENHAM, 2007; BEECHAM et al., 2008; BENAVIDES et al., 2010). A aplicação de Kitchenham (2004) sugere as seguintes etapas para o desenvolvimento da revisão: (i) questões de investigação; (ii) processos de pesquisa; (iii) critério para exclusão e inclusão, e avaliação da qualidade do estudo; (iv) coleta e análise de dados.

\section{(i) Questões de investigação}

Entre as questões de pesquisa que direcionaram este estudo estão: Quais são as instituições de pesquisa, os pesquisadores e os periódicos que desenvolvem e publicam estudos científicos sobre o tema de CM em alimentos? Quais são as aplicações e as barreiras de CM no setor alimentício?

\section{(ii) Processos de pesquisa}

Foi realizada uma pesquisa em cinco das principais bases de dados de periódicos científicos: Science Direct, Scopus, Emerald Insight, Proquest e Web of Science. A revisão sobre CM realizada por Fogliatto et al. (2012) utilizou os termos de busca "mass customization" e "mass customisation" para a busca de artigos. Neste estudo, utilizou-se os mesmos termos utilizados por Fogliatto et al. (2012), além dos termos isolados "customization" e "customisation", todos combinados com as palavras "food" ou "nutrition". A busca foi realizada nas bases de dados incorporando títulos, palavras-chaves e resumos. 
Uma revisão sistemática para identificação das possibilidades de aplicações e das barreiras de adoção da customização em massa na produção de alimentos

Calegari, L. P.; Fettermann, D. C.

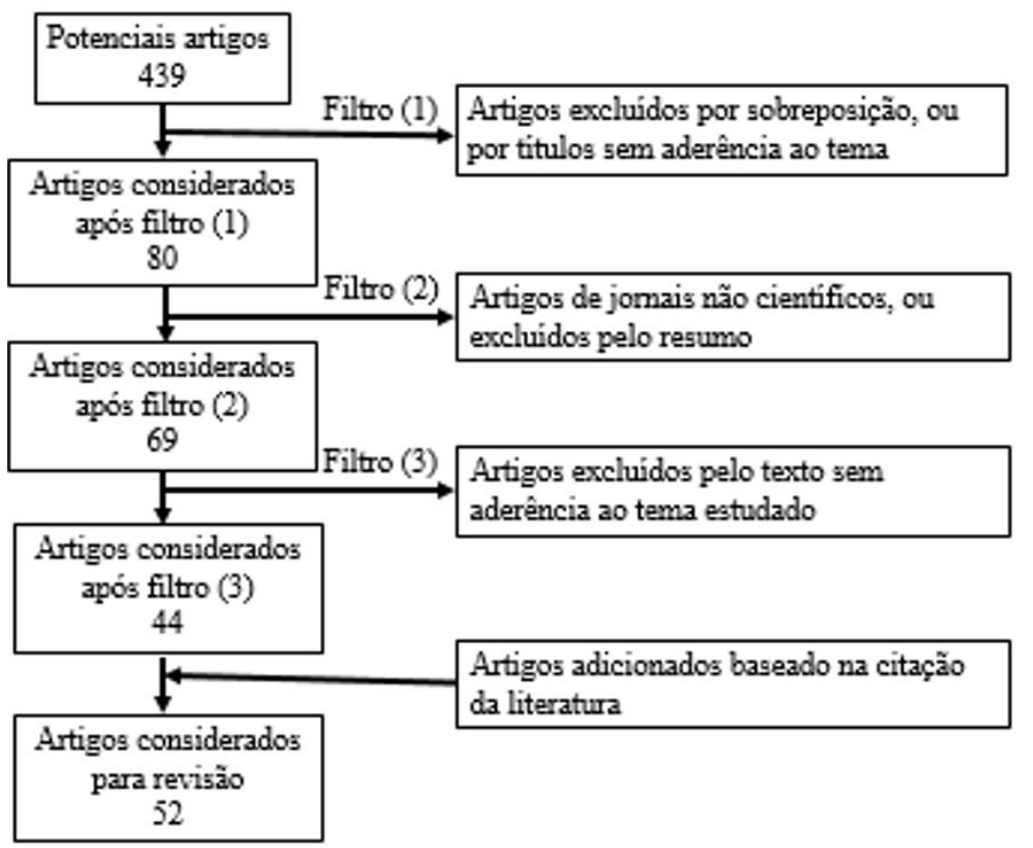

Figura 1. Procedimento para filtragem dos artigos considerados para esta revisão.

(iii)Critério para inclusão e exclusão, e avaliação de qualidade do estudo

O processo de busca identificou 439 artigos, que após o procedimento de filtragem resultou em 52 diferentes artigos analisados. Os procedimentos adotados durante esse filtro, conforme representado na Figura 1, excluíram artigos que não possuíam título, resumo ou texto aderente ao tema estudado. Os artigos selecionados não foram filtrados pela data de publicação, visto que a maior parte (49 artigos) dos artigos selecionados foram publicados a partir de 2000.

(iv) Coleta e análise de dados

São frequentes os estudos na literatura sobre CM que abordam os benefícios de sua aplicação (HART, 1995; KOTHA, 1995; SALVADOR et al., 2009), as barreiras ou dificuldades para a sua implementação (PINE, 1993; ÅHLSTRÖM; WESTBROOK, 1999; ZIPKIN, 2001) e os facilitadores que contribuem para o seu sucesso (SILVEIRA et al., 2001; PILLER; MÜLLER, 2004; FOGLIATTO et al., 2012). Seguindo este mesmo procedimento, foi realizada uma pesquisa para identificar nos artigos os benefícios, as barreiras e os facilitadores para a aplicação da CM no setor de alimentos. A coleta de dados foi realizada a partir da análise na íntegra dos artigos considerados na revisão. Além dos benefícios, barreiras e facilitadores para a aplicação da CM nos alimentos, também foram identificados nos artigos as aplicações em produtos alimentícios e os dados demográficos das publicações, tais como: periódicos, data de publicação e instituição dos autores. O procedimento foi realizado entre os meses de março de 2016 e janeiro de 2017. A seguir são apresentados os resultados obtidos a partir da revisão de literatura realizada.

\section{Resultados}

\subsection{Análise bibliométrica}

Como forma de analisar os temas abordados em cada publicação foram estabelecidas quatro diferentes categorias de temas de pesquisa. Na primeira categoria, denominada geral $(G)$, estão incluídos artigos que desenvolvem o objetivo de expor características e visões gerais de CM, incluindo o setor de alimentos. São artigos que apresentam estudos sobre o mercado alimentício, mencionam a indústria de alimentos ou utilizam dados do setor para compor o estudo. A segunda categoria de artigos, denominada processamento $(P)$, inclui temas direcionados para análise de características e alternativas de manufatura e processamento para customização de produtos alimentícios. A terceira categoria de artigos, denominada cliente $(C)$, incorpora o tema de análise da relação de $\mathrm{CM}$ com a interatividade do consumidor e sua percepção de valores sobre o produto alimentício customizado. Por fim, a quarta categoria de artigos, denominada cadeia de valor (V), é formada por estudos que focam na análise da relação de CM com a cadeia de suprimentos e logística da indústria alimentícia.

A partir da classificação dos artigos analisados nas categorias de temas ( $G$ - Geral; P - Processamento; C - Cliente; V - Cadeia de Valor), obteve-se uma representação cronológica da evolução do número de publicações em cada uma das categorias de estudos analisadas (Figura 1). A partir da Figura 2, pode-se observar que os trabalhos relacionados à análise da interação e percepção dos clientes para produtos alimentícios customizados (C) representam $42,30 \%$ do total de artigos publicados 
Uma revisão sistemática para identificação das possibilidades de aplicações e das barreiras de adoção da customização em massa na produção de alimentos

Calegari, L. P.; Fettermann, D. C.

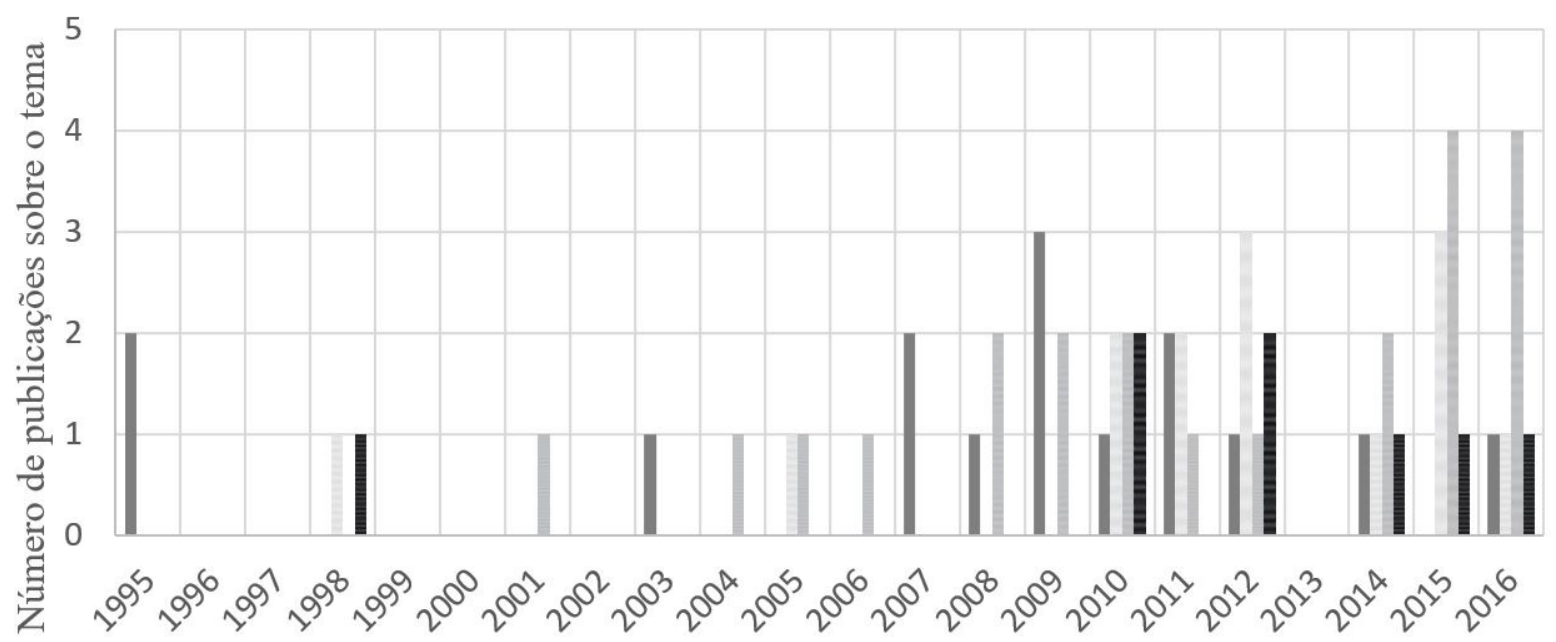

Anos

- Geral (G) $28,84 \%$
Cliente (C) $42,30 \%$

Processamento (P) 26,92\%

- Cadeia de Valor (V) 9,61\%

Figura 2. Representação cronológica da evolução dos temas.

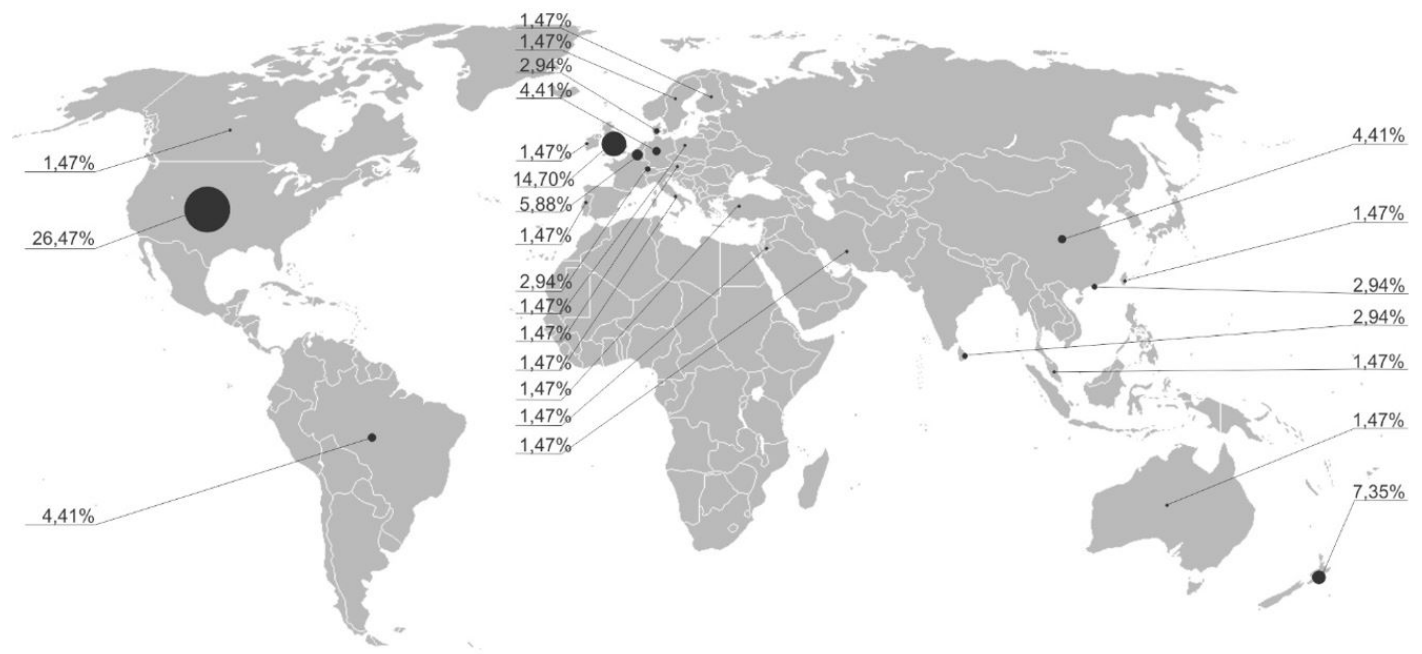

Legenda:

Local das instituições de origem dos autores dos artigos selecionados

Figura 3. Percentual do número de publicações sobre o tema por país.

durante o período analisado. As categorias de estudo apresentam uma dispersão no número de artigos ao longo do período analisado. A partir de 2015, foi verificado um crescimento na quantidade de publicações aderentes ao tema da análise da interação com clientes (C), que em 2015 e 2016 registraram a publicação de 4 artigos incluídos nesta revisão.

Com base nos dados obtidos pelo Google Scholar, os dois artigos considerados para esta pesquisa que apresentam maior quantidade de citações não possuem como temática principal a CM para a indústria de alimentos.
O artigo com maior citação, Grunert (2005), desenvolveu seu estudo para analisar a relação entre a qualidade, percepção de segurança e a demanda dos consumidores de produtos alimentícios. Foi considerado para esta revisão porque possui aderência com $\mathrm{CM}$, devido a sua análise da percepção dos consumidores, identificado como uma característica relevante a ser estudada na adoção de CM por uma empresa (SILVEIRA et al., 2001; FOGLIATTO et al., 2012). O trabalho de Fogliatto et al. (2012) apresenta uma revisão de literatura sobre $\mathrm{CM}$, incluindo estudos sobre sua aplicação no setor de alimentos (McINTOSH et al., 
Uma revisão sistemática para identificação das possibilidades de aplicações e das barreiras de adoção da customização em massa na produção de alimentos

Calegari, L. P.; Fettermann, D. C.

2010; BOLAND, 2008). Em sua revisão de literatura, Fogliatto et al. (2012) realizam uma análise sobre as barreiras e facilitadores para adoção da CM em diversos setores da indústria, sendo utilizada como referência de análise do presente estudo. Também pode ser verificado um crescente número de publicações ( $p$-valor $=0,0173$ ) sobre o tema de CM em produtos alimentícios, indicando a atualidade e uma tendência de crescimento nos estudos sobre o tema.

O periódico com maior quantidade de artigos integrantes desta revisão, o Trends in Food Science \& Technology, apresenta apenas três estudos. Periódicos com dois estudos incluídos na revisão são o Journal of Food Engineering, Journal of Retailing e International Journal of Hospitality Management. Os 43 demais estudos considerados nesta revisão foram publicados em outros diferentes periódicos, congressos ou livros. A análise dos periódicos confirma a dispersão dos estudos sobre CM em produtos alimentícios na literatura. São verificados periódicos de diversas áreas de concentração, com destaque para a área de gestão de operações e engenharia de alimentos. Quanto ao fator de impacto, verificamos que os periódicos direcionados para a área de alimentos apresentam média de seu fator de impacto $\left(\mathrm{JCR}_{\text {médio }}=2,80\right)$ superior em comparação com as demais áreas de estudo $\left(\mathrm{JCR}_{\text {médio }}=1,70\right)$.

Os centros de estudo sobre o tema da CM no setor de alimentos correspondem às instituições de filiação dos autores com publicações referenciadas nesta revisão de literatura. O número de publicações sobre o tema por país de filiação dos autores está representado na Figura 3. Verifica-se maior quantidade de publicações originadas dos Estados Unidos da América (18), seguido pelo Reino Unido (9) e Nova Zelândia (5), configurando-se nos países centro de estudos com maior quantidade de pesquisas sobre o tema.

Como forma de localizar mais especificadamente os centros de pesquisa que estudam a aplicação da CM em alimentos, foi identificada a instituição de filiação dos autores de cada um dos artigos analisados, juntamente com o país de filiação dos autores e a quantidade de artigos vinculados a estas instituições. Dentre essas instituições, podem ser destacadas com maior número de trabalhos publicados a University of Bath (3) e a Wageningen University (3), localizadas no Reino Unido e na Holanda, respectivamente.

Ao final da análise bibliométrica das publicações sobre CM em alimentos, foi possível constatar a dispersão dos periódicos das publicações, confirmando que o tema é estudado por diferentes áreas de conhecimento e não está consolidado na literatura. Também verificou-se um número crescente de publicações que abordam o tema, justificando sua atualidade, principalmente quando abordam a categoria de tema aderente à análise da interação com clientes. Por fim, foi verificada maior quantidade de estudos com autores vinculados a instituições dos Estados Unidos da América e Reino Unido, com destaque para a University of Bath (UK) e Wageningen University (HOL).

\section{Discussões}

\subsection{Aplicações de CM em alimentos}

A revisão sobre as aplicações da CM em alimentos considerada neste estudo identificou 40 aplicações em produtos alimentícios. Apesar de ser considerado um total de 52 artigos selecionados para o presente estudo, somente 21 artigos apresentaram aplicações focadas em produtos alimentícios específicos. Cada uma dessas aplicações foi categorizada por afinidade em seis grupos: (i) bebidas, (ii) condimentos, (iii) doces, (iv) fast foods, ( v) lácteos e ( $v i)$ refeições. Foi verificada maior frequência de estudos nos produtos de fast food (12) em detrimento das demais categorias de produtos. Essa maior concentração de aplicações em produtos de fast food pode ser explicada pela própria natureza desses produtos, que os tornam facilmente customizáveis, e pelo contato direto entre consumidor e manufatura, que contribui para a identificação das necessidades do cliente. Na Tabela 1 estão apresentados os produtos alimentícios mencionados na literatura de CM selecionada para o estudo:

A partir da Tabela 1, observa-se a presença de produtos que utilizam diferentes origens de insumos, evidenciado ainda a viabilidade de adoção de CM em produtos alimentícios que apresentem diferentes tipos de processamento. As aplicações que consideram a CM em bebidas foram desenvolvidas em sucos (FERREIRA; ALCÂNTARA, 2016), cervejas (DONADINI et al., 2016) e vinhos (VAN HOEK, 1999). Estes estudos apresentam aplicações diversas, tais como para o estudo da postergação da customização na cadeia de suprimentos (VAN HOEK, 1999; FERREIRA; ALCÂNTARA, 2016). Ainda referente à categoria bebidas, a literatura apresenta aplicações para a análise de percepção de valor do cliente diante diferentes combinações de ingredientes e diferentes mercados (DONADINI et al., 2016).

Para a categoria condimentos, o trabalho desenvolvido por Ferreira e Alcântara (2016) analisa a viabilidade de adotar postergação de cadeia em produtos derivados do tomate. Este estudo apresenta os diferentes tipos de postergação aplicados nas empresas que produzem produtos derivados de tomate, analisando como cada uma das possibilidades pode ser aplicada e o seu impacto no processo de produção. Também foi verificado o estudo sobre o comportamento do consumidor perante a customização de informações de rótulos de azeite de oliva extravirgem (BALCOMBE et al., 2016).

Na categoria "doces", foram verificados diversos produtos (tais como bolos, chocolates e biscoitos) 
Uma revisão sistemática para identificação das possibilidades de aplicações e das barreiras de adoção da customização em massa na produção de alimentos

Calegari, L. P.; Fettermann, D. C.

Tabela 1. Produtos alimentícios presentes na literatura de CM.

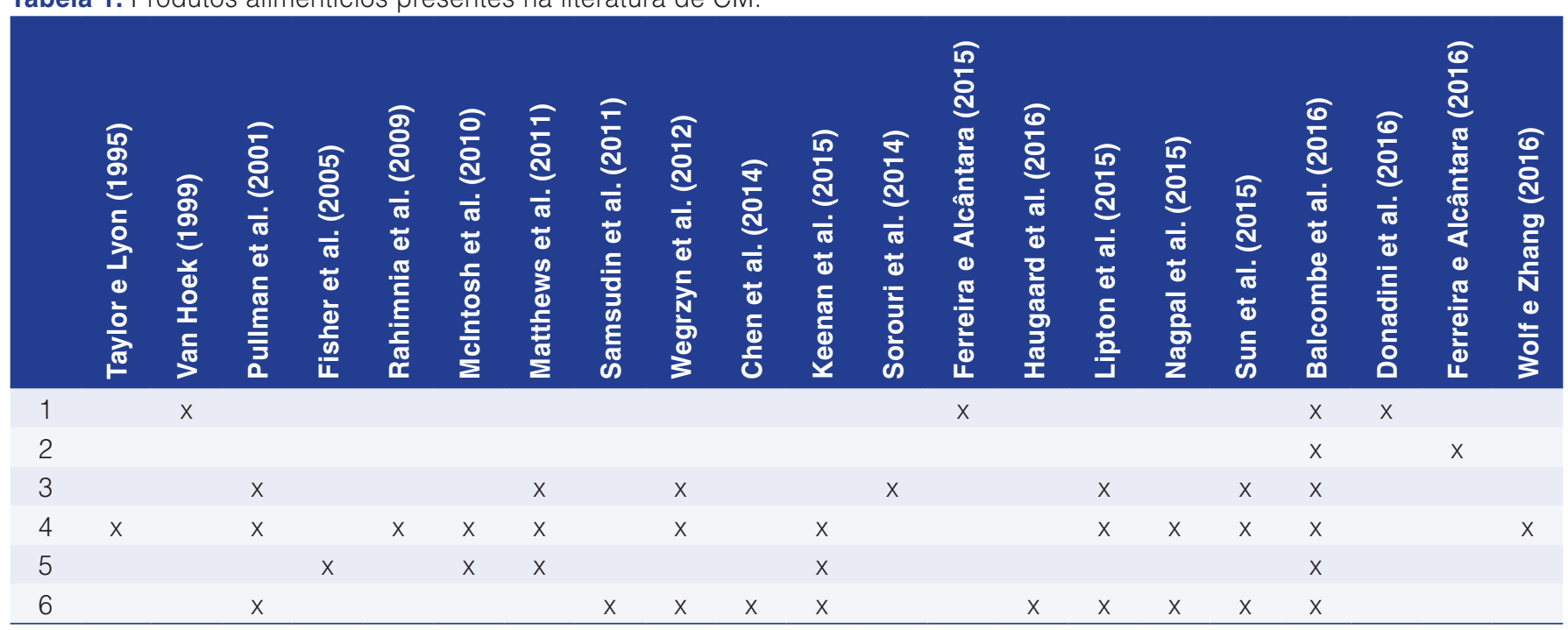

1 - Bebidas; 2 - Condimentos; 3 - Doces; 4 - Fast food; 5 - Lácteos; 6 - Refeições.

considerados em estudos para utilização de manufatura aditiva através da impressora 3D (WEGRZYN et al., 2012; LIPTON et al., 2015; SUN et al., 2015). Ainda para a categoria de doces, Sorouri et al. (2014) sugeriram a utilização de um sistema mecânico automatizado e inteligente para a fabricação de sorvetes.

Para a categoria dos alimentos "lácteos", foi identificada maior frequência de estudos com aplicações da CM em iogurtes (FISHER et al., 2005; McINTOSH et al., 2010; MATTHEWS et al., 2011). Estes estudos tiveram enfoque no levantamento de facilitadores e barreiras do processo de obtenção do produto, e de características dos produtos (tais como reologia) e do processo (tais como ciclo de maturação).

As categorias "fast food" "e "refeições" incluem vários produtos alimentícios diferentes, combinados entre si. Pode-se mencionar a aplicação de CM para a produção de pizzas (WEGRZYN et al., 2012; LIPTON et al., 2015; NAGPAL et al., 2015; SUN et al., 2015), cachorro-quente (RAHIMNIA et al., 2009; PULLMAN et al., 2001), hambúrguer (PULLMAN et al., 2001; WOLF; ZHANG, 2016), saladas (NAGPAL et al., 2015) e pratos prontos para restaurantes (SAMSUDIN et al., 2011; CHEN et al., 2014). Os estudos dessas categorias abordam principalmente a análise do comportamento dos consumidores frente a diferentes combinações de componentes de produtos (PULLMAN et al., 2001; NAGPAL et al., 2015; BALCOMBE et al., 2016; DONADINI et al., 2016; WOLF; ZHANG, 2016), ou para análise da interação dos consumidores com a customização de produtos (RAHIMNIA et al., 2009; SAMSUDIN et al., 2011; CHEN et al., 2014). Ainda para as categorias "fast food" $e$ "refeições", também foram identificados estudos propondo a utilização de manufatura aditiva, principalmente para a confecção de massas (WEGRZYN et al., 2012; LIPTON et al., 2015; SUN et al., 2015).

\subsection{Barreiras para adoção de CM em empresas alimentícias}

O desenvolvimento de novos produtos com viabilidade comercial exige que as possibilidades tecnológicas e de mercado sejam consideradas efetivamente no projeto do produto (ULRICH et al., 2003). A necessidade de atender a essas possibilidades tecnológicas, de mercado e gestão pode ser analisada na forma de barreiras (DOUGHERTY, 1992). O estudo sobre barreiras também pode ser denominado de outras formas na literatura, tais como fatores de sucesso (HOLLAND; LIGHT, 1999; SILVEIRA et al., 2001; FOGLIATTO et al., 2012) e dificuldades ou limites (ZIPKIN, 2001).

O estudo sobre as barreiras para implementação de CM pode ser encontrado em diversos estudos acerca do tema (PINE, 1993; MAcCARTHY et al., 2003; PILLER; MÜLLER, 2004; ZIPKIN, 2001; SALVADOR et al., 2009; DAABOUL et al., 2011). A literatura de CM direcionada ao setor alimentício incorpora novas barreiras para a adoção da CM nas empresas, principalmente em razão das particularidades provenientes do seu processamento (BOLAND, 2006; McINTOSH et al., 2010; MATTHEWS et al., 2011). Nos estudos sobre o tema, são mencionadas como barreiras para a implementação da CM na área de alimentos: (i) a perecibilidade dos alimentos, que considera a vida útil de um alimento (KOUKI et al., 2013; NEUSCHULZ et al., 2015); (ii) processamento, referente às restrições para produzir produtos alimentícios customizados (FISHER et al., 2005; MATTHEWS et al., 2011; McINTOSH et al., 2010); (iii) valores nutricionais, referentes à dificuldade em transcrever as características do produto customizado (GERMAN et al., 2004; BOLAND, 2008; BALCOMBE et al., 2016; REITBERGER et al, 2014); e (iv) percepção dos clientes, relacionada ao processo de agregação de valor 
Uma revisão sistemática para identificação das possibilidades de aplicações e das barreiras de adoção da customização em massa na produção de alimentos

Calegari, L. P.; Fettermann, D. C.

da customização do produto alimentício (PULLMAN et al., 2001; NAGPAL et al., 2015; WOLF; ZHANG, 2016).

A diferença nos processos da indústria em geral para a indústria alimentícia pode explicar a menor exploração da literatura sobre a CM no setor alimentício (BOLAND, 2008; McINTOSH et al., 2010; MATTHEWS et al., 2011). Enquanto para a indústria em geral o produto tem seu desempenho medido por meio de sua funcionalidade, para a indústria de alimentos são as características sensoriais (tais como textura e aparência) as relevantes para sua qualidade (FISHER et al., 2005; BOLAND, 2006). Todos os alimentos sofrem decomposição de forma natural, alterando as características químicas e físicas dos produtos alimentícios (BOLAND, 2008; McINTOSH et al., 2010; TRIENEKENS et al., 2012; MATTHEWS et al., 2011; SUN et al., 2015; FERREIRA; ALCÂNTARA, 2016).

As alterações químicas e físicas também podem ocorrer de forma induzida através de processos industriais, como os ciclos de maturação (McINTOSH et al., 2010; MATTHEWS et al., 2011), utilização de altas pressões (CAMPOS et al., 2003), técnicas de congelamento (RESENDE et al., 2002) e de processos térmicos (CALDERÓN-MIRANDA et al., 1998; MAHALIK; NAMBIAR, 2010). Esses processos são usualmente utilizados pelas indústrias alimentícias para o beneficiamento do produto, contribuindo também na eliminação de microrganismos (MAHALIK; NAMBIAR, 2010). As restrições sobre a forma de como o material alimentar pode ser processado sem prejuízo de sua qualidade (sabor, aparência e valores nutricionais) impõem limites para a utilização de tecnologia de processo (McINTOSH et al., 2010).

O estado físico (líquido, sólido, pastoso) ou a forma em que o alimento se encontra também podem afetar a customização do produto (MATTHEWS et al., 2011). Dessa maneira, a sensibilidade dos alimentos pode ser considerada uma barreira para o desenvolvimento e utilização de tecnologias que auxiliem a customização dos alimentos, tanto em automatizações para processos de transformações físico-químicas, quanto para operações de controle de produção (FISHER et al., 2005).

A crescente demanda por alimentos customizados (BOLAND, 2008) e o curto ciclo de vida que os produtos possuem, decorrente da rápida imitação pelos concorrentes (SUN, 2007; LEWIS et al., 2010), direcionam a indústria de processamento de alimentos a manter uma elevada variedade de produtos (FISHER et al., 2005). Tais características do mercado de produtos alimentícios customizados criam a necessidade de operações e sistemas de suprimentos flexíveis o suficiente para produzir maior quantidade de produtos alimentícios a um baixo custo (SALVADOR et al., 2009; LEWIS et al., 2010). Outras dificuldades também podem surgir devido a demandas alinhadas a períodos curtos ou sazonais (MATTHEWS et al., 2011). Em termos de sazonalidade, é preciso desenvolver alternativas para suprir as demandas do mercado em períodos entressafras. Para isso, a postergação de cadeia pode ser uma alternativa viável, como apresentado por Ferreira e Alcântara (2016) no caso de produções de derivados de tomates, que podem ser congelados em forma de polpa para serem utilizados em períodos em que a matéria-prima é escassa.

Análises realizadas por Stewart-Knox et al. (2013) sugerem que a nutrição personalizada é percebida como benéfica para a saúde humana. Há estudos que mencionam a possibilidade de aplicação de um conjunto de técnicas nutrigenômicas para estudar a relação entre o gene de cada indivíduo e o tipo de alimento recomendado (GERMAN et al., 2004; BOLAND, 2008), o que se tornaria importante para a caracterização de uma dieta personalizada (BOUHLAL et al., 2017). Quando as características genéticas de um consumidor são utilizadas como influenciadores do seu meio de nutrição, verifica-se um aumento da necessidade de customização, já que as dietas tendem a atender pedidos cada vez mais específicos devido à quantidade infinita de genomas. Esta necessidade de personalização demonstra, de certa forma, que as informações nutricionais compõem um fator que pode influenciar a avaliação de um produto, sua percepção e a intenção de compra (BURTON et al., 2009).

Neste caso, a personalização do produto pode dificultar a confecção da sua tabela nutricional em processos nos quais a previsão das quantidades de componentes não é precisa. Os consumidores de alimentos têm exigido produtos específicos, adaptados às suas necessidades e preferências individuais. Para a aceitação da customização de alimentos, os consumidores podem ser influenciados por atributos hedônicos e utilitários (NAGPAL et al., 2015); saúde e sabor que os alimentos apresentam (BHARADWAJ et al., 2009; TRIENEKENS et al., 2012); fatores ecológicos (GEHLHAR et al., 2009; HANKAMMER et al., 2016); sentimentos de promoção (WANG et al., 2013); nível de customização oferecida (WOLF; ZHANG, 2016); características culturais (PULLMAN et al., 2001; ADEIGBE et al., 2015; DONADINI et al., 2016); e também fatores como o tempo de espera e o preço do produto (PULLMAN et al., 2001).

Um dos requisitos para a adoção da CM é o entendimento do que o cliente realmente necessita. Algumas ferramentas são recomendadas para auxiliar os consumidores a identificar suas necessidades, minimizando complicações para suas decisões (MERTINS et al., 2012; SHABAH, 2015). Estas ferramentas contribuem para ajudar os clientes a perceberem a personalização do produto, para que identifiquem suas necessidades e traduzam-na em especificações de produto (SILVEIRA et al., 2001; PILLER; MÜLLER, 2004). Como exemplo, as tecnologias da rede sem fio combinada com os dispositivos móveis promovem uma infraestrutura para a integração e comunicação de 
Uma revisão sistemática para identificação das possibilidades de aplicações e das barreiras de adoção da customização em massa na produção de alimentos

Calegari, L. P.; Fettermann, D. C.

informações relacionadas ao pedido do produto customizado do cliente e a fabricação do alimento (SAMSUDIN et al., 2011; DORMAN et al., 2009). Em relação à utilização de interfaces gráficas para a personalização de produtos, os consumidores necessitam de um espaço de solução dentro do qual o consumidor possa agir criativamente, limitando a combinação das características do produto apenas para aqueles que possam ser efetivamente realizados, garantindo que o projeto de usuário seja possível (BIGLIARDI; GALATI, 2013).

\section{Conclusões}

A partir da revisão sistemática, foram obtidos 52 artigos considerados neste estudo. Como contribuições, esta pesquisa promoveu a reunião de informações que se encontram dispersas em diferentes áreas de estudo e que viabilizam a adoção de customização em massa (CM) para o setor alimentício (BOLAND, 2006; McINTOSH et al., 2010), identificando as principais dificuldades das empresas para a implementação de CM no setor. Como contribuição ao meio prático, foram obtidas considerações que podem auxiliar a adoção de CM para a produção de alimentos.

Ainda foram identificados diversos estudos com o objetivo de determinar o valor percebido pelo o cliente no alimento customizado (KEENAN et al., 2015; NAGPAL et al., 2015; WOLF; ZHANG, 2016; BALCOMBE et al., 2016). Apesar disso, o valor agregado ao cliente pela possibilidade de customizar produtos alimentícios ainda é pouco abordado nos estudos. Matthews et al. (2011) mencionam que alguns produtos são facilmente customizados pelos consumidores, sendo esta facilidade uma barreira para a adoção de CM pelas indústrias alimentícias. Dessa forma, o desenvolvimento de estudos sobre quais condições ocorrem agregação de valor ao produto alimentício customizado também se apresenta como oportunidade para futuras pesquisas.

Como resultado desta pesquisa, sugerimos como potenciais estudos futuros as seguintes questões: A identificação dos percepção de valor dos clientes frente à variedade de produtos disponibilizados pela estratégia de CM. Apesar de as tecnologias emergentes na área de alimentos permitirem maior variedade de opções para o cliente, nem sempre esta maior variedade agrega valor para o consumidor. Essas possibilidades de customização podem direcionar a uma maior agregação de valor. Entretanto, em um nível demasiadamente alto também podem confundir o cliente e reduzir o seu valor percebido, resultando no fenômeno denominado como mass confusion (CHEN; WANG, 2010). O estudo sobre o nível adequado de opções de alimentos se apresenta como oportunidade de pesquisa.

O desenvolvimento de rótulos e informações nutricionais sobre os alimentos customizados são uma dificuldade devido à diversidade de combinações possíveis para personalização de um produto alimentício. Estudos comprovam que a presença de informações sobre as características dos alimentos comercializados pode agregar valor ao produto, perante a percepção de valor dos consumidores (NAGPAL et al., 2015; BALCOMBE et al., 2016). Dessa forma, o desenvolvimento de estudos que identifiquem alternativas para possibilitar a customização de informações sobre as características dos alimentos também se caracteriza como oportunidade de pesquisa futuras. Alinhado a essas oportunidades, o estreitamento entre nutrição personalizada e estudos nutrigenômicos também poderiam ser abordados.

Pesquisas que alinhem tecnologias (como nanotecnologia e tecnologia genética) para processo de obtenção de produtos alimentícios, assim como estudos dos materiais que viabilizem processos aditivos, podem possibilitar a flexibilidade de processos alimentícios. A flexibilização dos processos é um facilitador para que a CM possa ser implementada.

\section{Referências}

ADEIGBE, R. T.; BALDWIN, S.; GALLION, K.; GRIER, S.; RAMIREZ, A. G. Food and beverage marketing to latinos: a systematic literature review. Health Education \& Behavior, v. 42, n. 5, p. 569-582, 2015. PMid:25504570. http://dx.doi. org/10.1177/1090198114557122.

ÅHLSTRÖM, P.; WESTBROOK, R. Implications of mass customization for operations management: an exploratory survey. International Journal of Operations \& Production Management, v. 19, n. 3 , p. 262-275, 1999. http://dx.doi.org/10.1108/01443579910249705.

ALFORD, D.; SACKETT, P.; NELDER, G. Mass customisation: an automotive perspective. International Journal of Production Economics, v. 65, n. 1, p. 99-110, 2000. http://dx.doi.org/10.1016/ S0925-5273(99)00093-6.

BALCOMBE, K.; FRASER, I.; LOWE, B.; MONTEIRO, DS. Information customization and food choice. American Journal of Agricultural Economics, v. 98, n. 1, p. 54-73, 2016. http:// dx.doi.org/10.1093/ajae/aav033.

BEECHAM, S.; BADDOO, N.; HALL, T.; ROBINSON, H.; SHARP, $\mathrm{H}$. Motivation in software engineering: a systematic literature review. Information and Software Technology, v. 50, n. 9, p. 860-878, 2008. http://dx.doi.org/10.1016/j.infsof.2007.09.004.

BENAVIDES, D.; SEGURA, S.; RUIZ-CORTÉS, A. Automated analysis of feature models 20 years later: a literature review. Information Systems, v. 35, n. 6, p. 615-636, 2010. http://dx.doi. org/10.1016/j.is.2010.01.001.

BHARADWAJ, N.; NAYLOR, R. W.; HOFSTEDE, F. Consumer response to and choice of customized versus standardized systems. International Journal of Research in Marketing, v. 26, n. 3, p. 216-227, 2009. http://dx. doi.org/10.1016/j.ijresmar.2009.04.001. 
Uma revisão sistemática para identificação das possibilidades de aplicações e das barreiras de adoção da customização em massa na produção de alimentos

Calegari, L. P.; Fettermann, D. C.

BIGLIARDI, B.; GALATI, F. Models of adoption of open innovation within the food industry. Trends in Food Science \& Technology, $v$. 30, n. 1, p. 16-26, 2013. http://dx.doi.org/10.1016/j.tifs.2012.11.001.

BOLAND, M. Innovation in the food industry: personalised nutrition and mass customisation. Innovation, v. 10, n. 1, p. 53-60, 2008. http://dx.doi.org/10.5172/impp.453.10.1.53.

BOLAND, M. Mass customisation of food. Journal of the Science of Food and Agriculture, v. 86, n. 1, p. 7-9, 2006. http://dx.doi. org/10.1002/jsfa.2348.

BOUHLAL, S.; MCBRIDE, C. M.; TRIVEDI, N. S.; AGURSCOLLINS, T.; PERSKY, S. Identifying eating behavior phenotypes and their correlates: a novel direction toward improving weight management interventions. Appetite, v. 111, n. 1, p. 142-150, 2017. PMid:28043857. http://dx.doi.org/10.1016/j.appet.2016.12.006.

BRABAZON, P. G.; MACCARTHY, B.; WOODCOCK, A.; HAWKINS, R. W. Mass customization in the automotive industry: comparing interdealer trading and reconfiguration flexibilities in order fulfillment. Production and Operations Management, v. 19, n. 5, p. 489-502, 2010. http://dx.doi.org/10.1111/j.19375956.2010.01132.x

BURTON, S.; HOWLETT, E.; TANGARI, A. H. Food for thought: how will the nutrition labeling of quick service restaurant menu items influence consumers' product evaluations, purchase intentions, and choices? Journal of Retailing, v. 85, n. 3, p. 258-273, 2009. http://dx.doi.org/10.1016/j.jretai.2009.04.007

CALDERÓN-MIRANDA, M. L.; GONZÁLEZ, M. F. S. M.; BARBOSACÁNOVAS, G. V.; SWANSON, B. G. Métodos no térmicos para procesamiento de alimentos: variables e inactivación microbiana. Brazilian Journal of Food Technology, v. 1, n. 1, p. 3-11, 1998.

CAMPOS, F. P.; DOSUALDO, G. L.; CRISTIANINI, M. The use of high pressure technology in food processing. Brazilian Journal of Food Technology, v. 6, n. 2, p. 351-368, 2003.

CHEN, P.; LIANG, Y. H.; LIN, T. C. Implementing a cooking and dietary management system using RFID technology. Mathematical Problems in Engineering, v. 2014, p. 234614, 2014. http:// dx.doi.org/10.1155/2014/234614

CHEN, Z.; WANG, L. Personalized product configuration rules with dual formulations: a method to proactively leverage mass confusion. Expert Systems with Applications, v. 37, n. 1, p. 383-392, 2010. http://dx.doi.org/10.1016/j.eswa.2009.05.050.

COHEN, D. L.; LIPTON, J. I.; CUTLER, M.; COULTER, D.; VESCO, A.; LIPSON, H. Hydrocolloid printing: a novel platform for customized food production. In: SOLID FREEFORM FABRICATION SYMPOSIUM, 20., 2009, Austin, TX. Proceedings..., Austin: University of Texas, 2009. p. 807-818.

COOK, D. J.; GREENGOLD, N. L.; ELLRODT, A. G.; WEINGARTEN, $S$. R. The relation between systematic reviews and practice guidelines. Annals of Internal Medicine, v. 127, n. 3, p. 210-216, 1997. PMid:9245227. http://dx.doi.org/10.7326/0003-4819-1273-199708010-00006.
DAABOUL, J.; CUNHA, C.; BERNARD, A.; LAROCHE, F. Design for mass customization: product variety vs. process variety. CIRP Annals-Manufacturing Technology, v. 60, n. 1, p. 169-174, 2011. http://dx.doi.org/10.1016/j.cirp.2011.03.093.

DAVIS, S. M. Future perfect. Reading: Addison-Wesley, 1987.

DONADINI, G.; FUMI, M. D.; KORDIALIK-BOGACKA, E.; MAGGI, L.; LAMBRI, M.; SCKOKAI, P. Consumer interest in specialty beers in three European markets. Food Research International, v. 85, p. 301-314, 2016. PMid:29544848. http://dx.doi.org/10.1016/j. foodres.2016.04.029.

DORMAN, K.; YAHYANEJAD, M.; NAHAPETIAN, A.; SUH, M. K.; SARRAFZADEH, M.; MCCARTHY, W.; KAISER, W. Nutrition monitor: a food purchase and consumption monitoring mobile system. In: INTERNATIONAL CONFERENCE ON MOBILE COMPUTING, APPLICATIONS, AND SERVICES, 35., 2009, San Diego. Proceedings..., Berlin Heidelberg: Springer, 2009. p. 1-11. https://doi.org/10.1007/978-3-642-12607-9_1.

DOUGHERTY, D. Interpretive barriers to successful product innovation in large firms. Organization Science, v. 3, n. 2, p. 179-202, 1992. http://dx.doi.org/10.1287/orsc.3.2.179.

FERREIRA, K. A.; ALCÂNTARA, R. L. C. Postponement application in orange juice companies: case studies. International Journal of Logistics Systems and Management, v. 22, n. 3, p. 331-349, 2015. http://dx.doi.org/10.1504/IJLSM.2015.072285.

FERREIRA, K. A.; ALCÂNTARA, R. L. C. Postponement adoption in manufacturers of tomato-derived products. British Food Journal, v. 118, n. 2, p. 362-378, 2016. http://dx.doi.org/10.1108/ BFJ-01-2015-0035.

FETTERMANN, D. C.; ECHEVESTE, M. E. S. New product development for mass customization: a systematic review. Production \& Manufacturing Research, v. 2, n. 1, p. 266-290, 2014. http://dx.doi.org/10.1080/21693277.2014.910715.

FISHER, C.; MULLINEUX, G.; MEDLAND, T.; HICKS, B. The design of food processing systems for improved responsiveness and late customisation. In: BRAMLEY, A.; BRISSAUD, D.; COUTELLIER, D.; MCMAHON, C. (Eds.). Advances in integrated design and manufacturing in mechanical engineering. Netherlands: Springer, 2005. p. 377-388. http://dx.doi.org/10.1007/1-4020-3482-2_30.

FOGLIATTO, F. S.; DA SILVEIRA, G. J. C.; BORENSTEIN, D. The mass customization decade: an updated review of the literature. International Journal of Production Economics, v. 138, n. 1, p. 14-25, 2012. http://dx.doi.org/10.1016/j.jpe.2012.03.002.

GEHLHAR, M. J.; REGMI, A.; STEFANOU, S. E.; ZOUMAS, B. $\mathrm{L}$. Brand leadership and product innovation as firm strategies in global food markets. Journal of Product and Brand Management, v. 18, n. 2, p. 115-126, 2009. http://dx.doi. org/10.1108/10610420910949013.

GERMAN, J. B.; YERETZIAN, C.; WATZKE, H. J. Personalizing foods for health and preference. Food Technology, v. 58, n. 12, p. 26-31, 2004. 
Uma revisão sistemática para identificação das possibilidades de aplicações e das barreiras de adoção da customização em massa na produção de alimentos

Calegari, L. P.; Fettermann, D. C.

GRUNERT, K. G. Food quality and safety: consumer perception and demand. European Review of Agriculture Economics, v. 32, n. 3, p. 369-391, 2005. http://dx.doi.org/10.1093/eurrag/jbi011.

HANKAMMER, S.; HORA, M.; CANETTA, L.; SEL, S. K. Userinterface design for individualization services to enhance sustainable consumption and production. Procedia CIRP, v. 47, p. 448-453, 2016. http://dx. doi.org/10.1016/j.procir.2016.03.077.

HART, C. W. L. Mass customization: conceptual underpinnings, opportunities and limits. International Journal of Service Industry Management, v. 6, n. 2, p. 36-45, 1995. http://dx.doi. org/10.1108/09564239510084932

HAUGAARD, P.; BROCKHOFF, P. B.; LÄHTEENMÄKI, L. Objective measures of meal variety lacking association with consumers' perception of variety with self-selected buffet meals at work. Food Quality and Preference, v. 51, p. 123-129, 2016. http:// dx.doi.org/10.1016/j.foodqual.2016.03.004

HOLLAND, C. P.; LIGHT, B. A critical success factors model for ERP implementation. IEEE Software, v. 16, n. 3, p. 30-36, 1999. http://dx.doi.org/10.1109/52.765784

KEENAN, G. S.; BRUNSTROM, J. M.; FERRIDAY, D. Effects of meal variety on expected satiation: evidence for a 'perceived volume' heuristic. Appetite, v. 89, p. 10-15, 2015. PMid:25599925. http://dx.doi.org/10.1016/j.appet.2015.01.010.

KITCHENHAM, B. Procedures for performing systematic reviews. Vol. 33. Keele: Keele University, 2004.

KITCHENHAM, B.A. Guidelines for performing systematic literature reviews in software engineering. Keele: EBSE, 2007. (EBSE Technical Report 2.3).

KOTHA, S. Mass customization: implementing the emerging paradigm for competitive advantage. Strategic Management Journal, v. 16, n. S1, p. 21-42, 1995. http://dx.doi.org/10.1002/ smj. 4250160916 .

KOUKI, C.; SAHIN, E.; JEMAI, Z.; DALLERY, Y. Assessing the impact of perishability and the use of time temperature technologies on inventory management. International Journal of Production Economics, v. 143, n. 1, p. 72-85, 2013. http:// dx.doi.org/10.1016/j.ijpe.2010.09.032

LEWIS, M.; BRANDON-JONES, A.; SLACK, N.; HOWARD, M. Competing through operations and supply: the role of classic and extended resource-based advantage. International Journal of Operations \& Production Management, v. 30, n. 10, p. 10321058, 2010. http://dx.doi.org/10.1108/01443571011082517.

LINDEN, F. J.; SCHMID, K.; ROMMES, E. Software product lines in action: the best industrial practice in product line engineering. Secaucus: Springer, 2007

LIPTON, J. I.; CUTLER, M.; NIGL, F.; COHEN, D.; LIPSON, H. Additive manufacturing for the food industry. Trends in Food Science \& Technology, v. 43, n. 1, p. 114-123, 2015. http:// dx.doi.org/10.1016/j.tifs.2015.02.004.

MACCARTHY, B.; BRABAZON, P. G.; BRAMHAM, J. Fundamental modes of operation for mass customization. International Journal of Production Economics, v. 85, n. 3, p. 289-304, 2003. http:// dx.doi.org/10.1016/S0925-5273(03)00117-8

MAHALIK, N. P.; NAMBIAR, A. N. Trends in food packaging and manufacturing systems and technology. Trends in Food Science \& Technology, v. 21, n. 3, p. 117-128, 2010. http:// dx.doi.org/10.1016/j.tifs.2009.12.006.

MATTHEWS, J.; MCINTOSH, R.; MULLINEUX, G. Contrasting opportunities for mass customisation in food manufacture and food processes. In: FOGLIATTO, F. S.; SILVEIRA, G. J. C. Mass customization. London: Springer, 2011. p. 353-374. http://dx.doi. org/10.1007/978-1-84996-489-0_17.

MATTHEWS, J.; SINGH, B.; MULLINEUX, G.; MEDLAND, T. Constraint-based approach to investigate the process flexibility of food processing equipment. Computers \& Industrial Engineering, v. 51, n. 4, p. 809-820, 2006. http://dx.doi. org/10.1016/j.cie.2006.09.003.

MCINTOSH, R. I.; MATTHEWS, J.; MULLINEUX, G.; MEDLAND, A. $J$. Late customisation: issues of mass customisation in the food industry. International Journal of Production Research, v. 48, n. 6 , p. 1557-1574, 2010. http://dx.doi.org/10.1080/00207540802577938.

MERTINS, K.; JAEKEL, F.; DENG, Q. Towards information customization and interoperability in food chains. In: International IFIP WORKING CONFERENCE ON ENTERPRISE INTEROPERABILITY, 122., 2012, Harbin, China. Proceedings... Berlin Heidelberg: Springer, 2012. p. 92-103. http://dx.doi.org/10.1007/978-3-642-33068-1_10.

NAGPAL, A.; LEI, J.; KHARE, A. To choose or to reject: the effect of decision frame on food customization decisions. Journal of Retailing, v. 91, n. 3, p. 422-435, 2015. http://dx.doi.org/10.1016/j. jretai.2014.12.001

NEUSCHULZ, E. L.; MUELLER, T.; BOLLMANN, K.; GUGERLI, F.; BÖHNING-GAESE, K. Seed perishability determines the caching behaviour of a food-hoarding bird. The Journal of animal ecology, v. 84, n. 1, p. 71-78, 2015. PMid:25156134. http://dx.doi.org/10.1111/1365-2656.12283.

PETERS, L.; SAIDIN, H. IT and the mass customization of services: the challenge of implementation. International Journal of Information Management, v. 20, n. 2, p. 103-119, 2000. http:// dx.doi.org/10.1016/S0268-4012(99)00059-6.

PILLER, F. T.; MÜLLER, M. A new marketing approach to mass customisation. International Journal of Computer Integrated Manufacturing, v. 17, n. 7, p. 583-593, 2004. http://dx.doi.org/ 10.1080/0951192042000273140

PILLER, F.; SCHUBERT, P.; KOCH, M.; MOSLEIN, K. Overcoming mass confusion: collaborative customer co design in online communities. Journal of Computer-Mediated Communication, v. 10, n. 4, p. 1-10, 2005. http://dx.doi.org/10.1111/j.1083-6101.2005. tb00271.x.

PINE, B. J. Mass customization: the new frontier in business competition. Harvard: Harvard Business Press, 1993.

PIZAM, A. Does the tourism/hospitality industry possess the characteristics of a knowledge-based industry? International 
Uma revisão sistemática para identificação das possibilidades de aplicações e das barreiras de adoção da customização em massa na produção de alimentos

Calegari, L. P.; Fettermann, D. C.

Journal of Hospitality Management, v. 26, n. 4, p. 759-763, 2007. http://dx.doi.org/10.1016/j.ijhm.2007.05.001.

PULLMAN, M. E.; VERMA, R.; GOODALE, J. C. Service design and operations strategy formulation in multicultural markets. Journal of Operations Management, v. 19, n. 2, p. 239-254, 2001. http://dx.doi.org/10.1016/S0272-6963(00)00059-0.

RAHIMNIA, F.; MOGHADASIAN, M.; CASTKA, P. Benchmarking leagility in mass services: the case of a fast food restaurant chains in Iran. Benchmarking, v. 16, n. 6, p. 799-816, 2009. http://dx.doi.org/10.1108/14635770911000123.

REIM, W.; PARIDA, V.; ÖRTQVIST, D. Product-Service Systems (PSS) business models and tactics: a systematic literature review. Journal of Cleaner Production, v. 97, p. 61-75, 2015. http:// dx.doi.org/10.1016/j.jclepro.2014.07.003.

REITBERGER, W.; SPREICER, W.; FITZPATRICK, G. Situated and mobile displays for reflection on shopping and nutritional choices. Personal and Ubiquitous Computing, v. 18, n. 7, p. 1721-1735, 2014. http://dx.doi.org/10.1007/s00779-014-0781-4.

RESENDE, J. V.; SILVEIRA JUNIOR, V.; NEVES FILHO, L. C. Air blast freezing of fruit pulp models in commercial boxes: influence of preferential channels in the bed on freezing times estimating. Food Science and Technology, v. 22, n. 3, p. 319-327, 2002. http://dx.doi.org/10.1590/S0101-20612002000300021.

SALVADOR, F.; HOLAN, P. M.; PILLER, F. T. Cracking the code of mass customization. MIT Sloan Management Review, v. 50, n. 3, p. 71-78, 2009.

SAMSUDIN, N. A.; KHALID, S. K. A.; KOHAR, M. F. A. M.; SENIN, Z.; IHKASAN, M. N. A customizable wireless food ordering system with realtime customer feedback. In: IEEE SYMPOSIUM ON WIRELESS TECHNOLOGY AND APPLICATIONS (ISWTA), 2011, Langkawi, Malaysia. Proceedings..., New York: IEEE, 2011. p. 186-191. http://dx.doi.org/10.1109/ISWTA.2011.6089405.

SHABAH, A. HUMANIT3D for disaster response: an assessment of mass customization on organizational performance under turbulent environments. Procedia Engineering, v. 107, p. 223-236, 2015. http://dx.doi.org/10.1016/j.proeng.2015.06.077.

SILVEIRA, G.; BORENSTEIN, D.; FOGLIATTO, F. S. Mass customization: literature review and research directions. International Journal of Production Economics, v. 72, n. 1, p. 1-13, 2001. http://dx.doi.org/10.1016/S0925-5273(00)00079-7.

SOROURI, M.; VYATKIN, V.; SALCIC, Z. MIRA: Enabler of mass customization through agent-based development of intelligent manufacturing systems. In: IEEE INTERNATIONAL CONFERENCE ON ROBOTICS AND AUTOMATION (ICRA). Hong Kong, China, 2014. Proceedings... New York: IEEE, 2014. p. 258-263. http:// dx.doi.org/10.1109/ICRA.2014.6906619.

STEWART-KNOX, B.; KUZNESOF, S.; ROBINSON, J.; RANKIN, A.; ORR, K.; DUFFY, M.; POÍNHOS, R.; DE ALMEIDA, M. D. V.; MACREADY, A.; GALLAGHER, C.; BEREZOWSKA, A.; FISCHER, A. R. H.; NAVAS-CARRETERO, S.; RIEMER, M.; TRACZYK, I.; GJELSTAD, I. M. F.; MAVROGIANNI, C.; FREWER, L. J. Factors influencing European consumer uptake of personalised nutrition.
Results of a qualitative analysis. Appetite, v. 66, p. 67-74, 2013. PMid:23500415. http://dx.doi.org/10.1016/j.appet.2013.03.001.

SUN, D.W. Future of food engineering. Journal of Food Engineering, v. 83, n. 1, p. 1-2, 2007. http://dx.doi.org/10.1016/j. jfoodeng.2007.03.001.

SUN, J.; PENG, Z.; ZHOU, W.; FUH, J. Y. H.; HONG, G. S.; CHIU, A. A review on $3 D$ printing for customized food fabrication. Procedia Manufacturing, v. 1, p. 308-319, 2015. http://dx.doi. org/10.1016/j.promfg.2015.09.057

TAYLOR, S.; LYON, P. Paradigm lost: the rise and fall of McDonaldization. International Journal of Contemporary Hospitality Management, v. 7, n. 2/3, p. 64-68, 1995. http:// dx.doi.org/10.1108/09596119510080024.

TRIENEKENS, J. H.; WOGNUM, P. M.; BEULENS, A. J.; VAN DER VORST, J. G. Transparency in complex dynamic food supply chains. Advanced Engineering Informatics, v. 26, n. 1, p. 55-65, 2012. http://dx.doi.org/10.1016/j.aei.2011.07.007.

TSENG, M. M.; JIAO, J.; MERCHANT, M. Design for mass customization. CIRP Annals-Manufacturing Technology, V. 45, n. 1, p. 153-156, 1996. http://dx.doi.org/10.1016/S00078506(07)63036-4

ULRICH, P. V.; ANDERSON-CONNELL, L. J.; WU, W. Consumer co-design of apparel for mass customization. Journal of Fashion Marketing and Management: An International Journal, v. 7, n. 4, p. 398-412, 2003. http://dx.doi.org/10.1108/13612020310496985.

VAN HOEK, R. I. Postponement and the reconfiguration challenge for food supply chains. Supply Chain Management, v. 4, n. 1, p. 18-34, 1999. http://dx.doi.org/10.1108/13598549910255068.

VERDOUW, C.; BEULENS, A.; WOLFERT, S. Towards software mass customization for business collaboration. In: ANNUAL SRII GLOBAL CONFERENCE. San Jose, 2014. Proceedings... New York: IEEE, 2014. p. 106-115. http://dx.doi.org/10.1109/ SRII.2014.24.

WANG, Y.; KANDAMPULLY, J.; JIA, H. "Tailoring" customization services: Effects of customization mode and consumer regulatory focus. Journal of Service Management, v. 24, n. 1, p. 82-104, 2013. http://dx.doi.org/10.1108/09564231311304206.

WEDZICHA, B.; ROBERTS, C. Modelling: a new solution to old problems in the food industry. Food Manufacturing Efficiency., v. 1, n. 1, p. 1-7, 2006. http://dx.doi.org/10.1616/fme.2006.1.1.1.

WEGRZYN, T. F.; GOLDING, M.; ARCHER, R. H. Food layered manufacture: a new process for constructing solid foods. Trends in Food Science \& Technology, v. 27, n. 2, p. 66-72, 2012. http://dx.doi.org/10.1016/j.tifs.2012.04.006.

WOLF, A.; ZHANG, L. The effect of customization and gender on customers' attitude. International Journal of Hospitality Management, v. 56, p. 28-32, 2016. http://dx.doi.org/10.1016/j. ijhm.2016.04.001.

ZIPKIN, P. The limits of mass customization. MIT Sloan Management Review, v. 42, n. 3, p. 81, 2001. 